\title{
Psychology in the ICT Era: Electronic Psychology
}

\author{
Athanasios Drigas, Leyteris Koukianakis, and Yannis Papagerasimou \\ NCSR 'Demokritos', \\ Institute of Informatics and Telecommunications, \\ Net Media Lab, \\ Agia Paraskevi, 153 10, Athens, Greece \\ $\{d r$, kouk, ypapa $\}$ @imm. demokritos.gr
}

\begin{abstract}
Cognitive science is the scientific domain which studies, analyses, simulates and infers for various aspects, functions and procedures of human mentality such as, thinking, logic, language, knowledge, memory, learning, perception and the ability to solve problems. E-psychology is in a close relation with the cognitive science domain, but expands beyond it, as e-psychology is the efficient convergence of Psychology and Information and Communication Technologies (ICTs). E-psychology offers a number of services such as supporting, diagnosis, assessment, therapy, counseling, intervention and tests through an effective exploitation of ICTs. This article presents a user-friendly, flexible and adaptive electronic platform, which supports both synchronous and asynchronous e-psychology activities through the use of informative and communicative tools and services, which can be adapted to support various methods of e-psychology activities. It is important to underline that e-psychology is not an alternative psychology field, but a resource to enhance the conventional psychology process.
\end{abstract}

Keywords: E-psychology, ICTs, cognitive science.

\section{Introduction}

The rapid advance of ICTs and the Internet over the course of the last fifteen years has affected a significant number of aspects of contemporary life including education. Nowadays, educational e-content can be found anywhere, anytime and to anyone who can connect to the Internet. Hence, it was only natural for universities and academic institutions to use this development to their advantage by providing on-demand web based education and training through course delivery platforms such as the Ariadne Web based learning environment and electronic textbooks through the use of authoring tools such as InterBook [1], [2].

A large number of sciences including Psychology have taken advantage and have exploited the numerous capabilities of the Internet to their benefit. More particularly, psychology uses the Internet in order to create programs for psychological intervention, assessment, orientation, and specialized counseling, as a means of prevention. The Internet not only constitutes a new communicative medium between patient and therapist but is also the future of psychology [4], [5], [6], [7], [8], [9], [10], [11]. This new virtual environment uses such tools as e-mail, chat rooms, discussion forums and 
audio and video conference for the communication and interaction of the therapist with the patient, tools which are also used for educational purposes in e-learning environments [12], [13].

It is common knowledge that the Internet is packed with information of uncertain quality and prestige. Taking this fact into account, it becomes more than apparent that it is absolutely essential to know how and where to extract useful and qualitative information from, regarding the object of ones interest. This becomes even more important and more vital when the object of interest regards health issues [14], [15], [16], [17]. According to a recent study, real patients and supported individuals were found to trust entirely and resort to prominent and well-known websites in order to gather information regarding their health issue, without prior guidance by neither their therapists nor even by Internet experts. That is, they visited websites of major hospitals, health organizations as well as of government organizations in order to acquire information [3]. Quality information with substantial validity and weight can have a positive psychological effect on patients. Hence, it becomes crucial that psychologists embed this new and innovative means of psychology in their practice and view it as a means to enhance the entire psychology process [18], [19].

Based on the aforementioned framework, an e-psychology platform was developed which was also based on the following principles. Firstly, it was decided that since the e-psychology platform is partially but principally addressed to supported individuals, which is a very sensitive social group, it was essential that the design was as userfriendly and user-centered as possible and according to the user needs. Secondly, it was decided that the electronic content and the tools of the platform should be modular and flexible. With this modularity and flexibility of both the e-content and the tools, the administrator has the capability to provide environments and services of different types. This is done, in order for the platform to support different target groups, various categories of supported individuals (depending on the type of support they are receiving) and different categories of scientists (psychologists training, staff training) on the one hand and on the other, to support different psychological procedures. For instance, reusability can support a procedure that is based on behavioral psychological principles or it can organize an environment that is based on and embeds the diagnostic principles and tools of cognitive psychology. Finally, the instructional methods that are embedded within the developed web-based platform use most of the available modern multimedia and communicative technologies of the Internet and offer various modes for the delivery and presentation of the electronic content.

\section{E-Psychology: An Overview}

\subsection{Modelling Psychology Procedures}

The first step that is required in order to develop and design an e-psychology environment is to model the operations and procedures of the psychology cycle (Fig. 1). The most important step in the procedure of psychological services provision is diagnosis. Diagnosis, as it is presented below is based on the one hand on the diagnostic criteria, which are associated with DSM-IV and ICD-10, while on the other hand it utilizes and is based on diagnostic instruments such as interview, psychometric tests and observation. The result of the above is the production of the assessment report. 


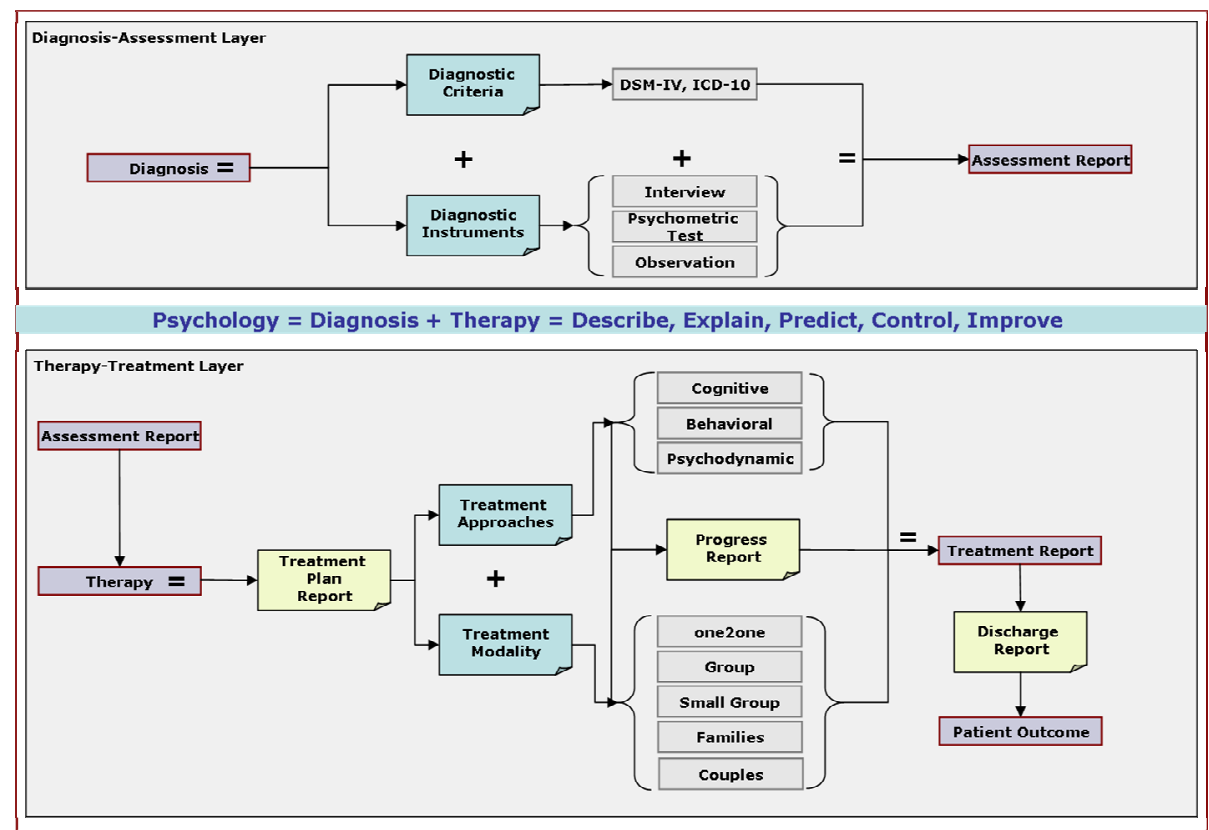

Fig. 1. Psychology model diagram

It is obvious that these distinguished steps of the diagnosis procedure are the main structural ingredients with which it is possible to create an embedded system in an e-psychology environment, which performs the operations of diagnosis support (e-diagnosis).

Following the modeling of diagnosis, it is very important to underline the importance of modeling the stages and the procedures of therapy - treatment to the process of designing and realizing an e-psychology environment.

Based on the assessment report that was derived through the diagnosis process, the therapy - treatment process follows the stages that are depicted above. The basis for the development of these stages for this process is the treatment plan report, which consists of the treatment approaches and the treatment modality. With the term treatment approaches we refer to the various therapeutic and theoretical approaches of psychology such as cognitive, behavioral, psychodynamic and existential. On the other hand, with the term treatment modality we refer to the various means of intervention such as one to one, small group, family and couples.

Finally, at the last stage of the modeled therapeutic process, the treatment report is produced as well as the discharge report and finally the patient outcome.

\subsection{E-Psychology Process Cycle}

Following the discussion about modeling psychology procedures, this chapter presents the e-psychology process cycle (Fig. 2), which is realized in our platform and which was the basis for the development of the e-psychology environment. 
The Supported Person (SP) has access through a registration form over a Secure Socket Layer (SSL) to the registration department and his/her application becomes accepted after the necessary validation process. Following this, a temporary supported person page is created and through certain electronic procedures such as psychometric tests and interviews the assessment report is produced.

Furthermore, through the creation of the supported person's history folder and the appropriate review, the supported person's personal information is inserted in the appropriate virtual clinic department where suitable treatment modalities are chosen and applied through suitably designed treatment plans. At this stage, the informative tools that support and realize the aforementioned procedures are the Supported Person calendar, health files, personal information, psychologist, health library and forum.

At the end of the e-psychology process cycle there is the patient e-outcome provided that all the necessary conditions of the treatment plan report, progress report and discharge report are met successfully.

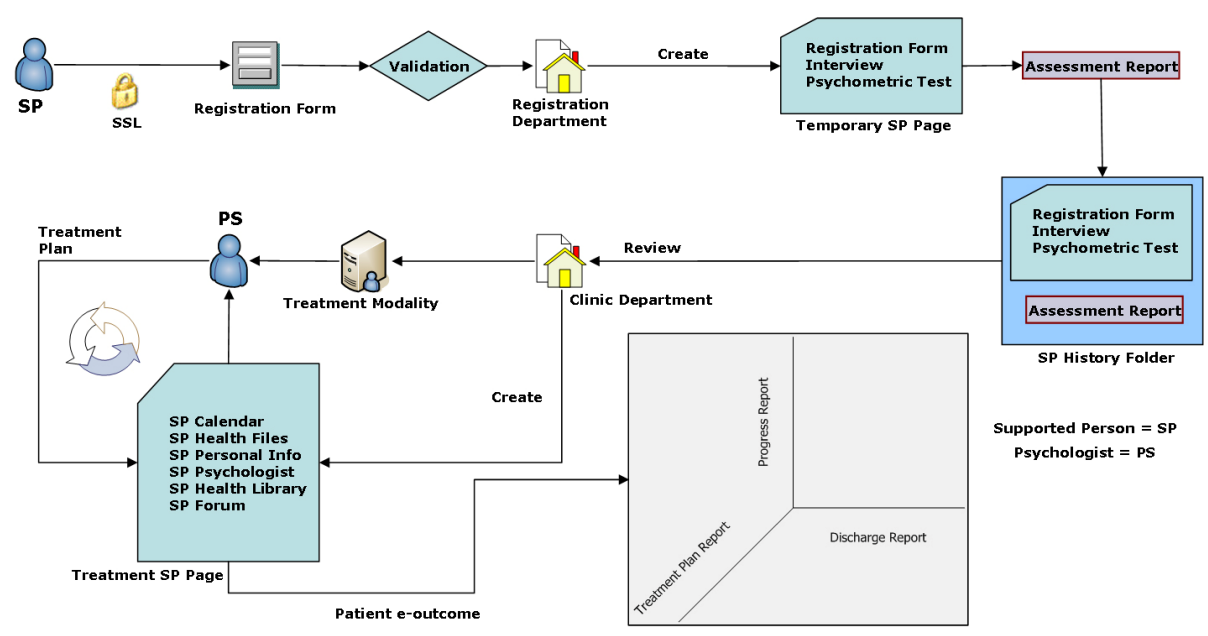

Fig. 2. E-psychology process cycle diagram

\subsection{E-Psychology and E-Learning Tools}

The presented e-psychology process cycle is realized through special information and communication technologies tools and services. These are divided into two categories: the informative and the communicative tools and services. The latter are divided into two subcategories: the synchronous (real-time) and the asynchronous (non realtime) tools and services, which determine the terms synchronous and asynchronous environments. These tools and services were used for the development of the epsychology platform imprinting the traditional learning and psychology processes with synchronous and asynchronous learning and psychology tools in the platform.

In addition, the user levels and user interfaces of the e-psychology platform comprise the administrator, the therapist - psychologist, the patient - supported person and finally the visitor who support similar actions with the e-learning user levels namely, 
administrator, instructor, student and user. Finally, the seminars, classes, courses and educational material from the e-learning circle, are supported by the same tools in similar procedures with the therapeutic entities, small groups, therapeutic process, and supporting material, from the e-psychology circle.

The specifications of the user levels, the e-content and the e-tools in an epsychology platform can be easily implemented through a simple correspondence of the psychology ontologies to the generic e-learning ontologies.

E-psychology and e-learning have a very close relationship. It is obvious that the roles of the "instructor" and the "student" are transformed into the roles of the "psychologist" and the "supported individual" respectively. The "classes" are transformed into "small therapy groups" and the "courses" into "supporting material". The "consulting material" (examples, exercises, multiple choice tests) correspond respectively to diagnosis, educational exercise for treatment and diagnostic tests. Finally, all the tools (web directory, glossary, references, video and audio lectures, events calendar, news, announcements, mailing lists, e-library, message box, email, video and audio conference, discussion forums, instant messaging, chat, and telephony) can be easily applied to both platforms.

\section{E-Psychology Platform Presentation}

\subsection{E-Psychology Platform Structure and User Levels}

Based on the discussion in chapter 2, we have developed an e-psychology platform which is abstractly depicted in (Fig. 3). The environment supports the operations of ediagnosis and e-therapy that lead to the successful coverage of the aims that were set according to the followed psychological approach, in order to finally reach the eoutcome (provided that the aims are met successfully).

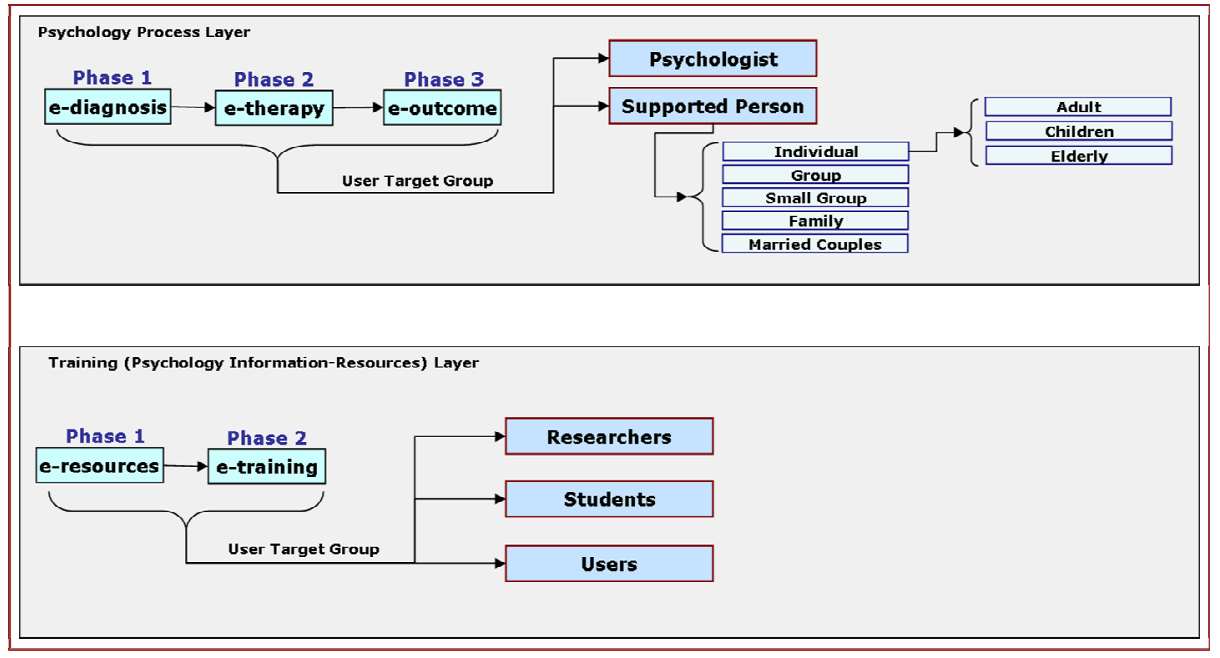

Fig. 3. Abstract presentation of the e-psychology platform structure 
These operations support and refer to the user target groups. These comprise the supported persons on the one hand and on the other the therapists, who receive support through the e-psychology environment in order to execute their task in the best possible way. The supported persons comprise individuals, groups, small groups, families and couples while the individuals can be either adults, children or elderly.

The module that refers to training plays a very important role in the structure of the e-psychology platform. Training is a very important tool in the e-psychology field as it is used not only in the various fields of psychology for the support of the supported persons, but also for the support and constant upgrade of the cognitive skills of the therapists and the formation of the way that they treat both the supported persons as well as the various procedures within the e-psychology environment.

Beyond the therapists and supported persons, the training module of the epsychology platform constitutes a very powerful tool for the training of researchers and students who are given permission to access the e-psychology platform. Finally, the importance of the training modules' role in the training of the common user (mean citizen) should not be overlooked as it informs and trains him/her on various interesting subjects regarding everyday psychological health such as stress control, possible perception or even behavioral deflections in children, memory issues with adults and the elderly and finally, substances abuse etc.

The e-psychology platform comprises four different user levels namely administrator, psychologist - therapist, patient - supported individual and visitor. Each of these user levels has a different role and different permission levels to both informative and communicative tools and services of the platform.

The role of the administrator is to manage the e-platform using the administrative tools of the system. These tools give the administrator the power to hand permission to the other users of the platform to access the various tools and services, depending on their needs. Finally, the administrator is in constant communication with the therapist - psychologist regarding their sessions with patients and their progress.

Moreover, the role of the therapist - psychologist is to create and organize the econtent of the platform in terms of its delivery to the patients - supported individuals as well as to anyone who is interested in viewing it. In particular, general information regarding psychological issues is uploaded to the system but more importantly, the electronic content of the sessions that the therapist engages in with a patient. The scope of this is to enable the patients to revise the session in their own time and have a better perception of it. Finally, there is also the online communication, which aims at the further interaction of the two parts and the reply of questions that may rise.

Furthermore, the role of the patients - supported individuals is that of the regulator of the e-platform. That is to say that it is the patients that view the e-content, engage in the online sessions with the therapists and hence it is up to them to determine whether the platform is worth while or not. Additionally, the patients have full access to all the information and the services provided by this e-psychology platform.

Finally, the visitor (common citizen) has the unique opportunity to access the econtent of the e-psychology platform. This means access to consulting material, general sessions and discussion forums where the visitor can go through discussions between therapists and patients for purely informative and educational purposes. This innovation is in accord with the principle 'information for all' which conforms to the knowledge society strategies. It must be noted that these discussions are generic and 
non confidential. It is obvious that visitors do not have the ability to access the econtent that has to do with confidential sessions and discussions between therapists and patients. This personal information is protected and can only be accessed by the other three user levels who are authorized to do so.

\section{Conclusions}

The incorporation of ICTs as well as of Internet technologies within the traditional psychology process results in what is commonly known as e-psychology, which was the topic of this article. Although e-psychology is an innovation and a step forward for traditional psychology, it was underlined from the start that under no circumstances does it substitute, replace or undermine the traditional psychology process but acts more as a means to enhance it and to complement it.

E-psychology in its broad sense enables the therapists - psychologists as well as the patients - supported individuals to use and incorporate technology in their sessions bringing the latter to a whole new level. While carrying on their traditional sessions both parts have the opportunity to experiment simultaneously with this new electronic process in order to deduce useful conclusions and to make the whole experience of a virtual session more appealing and beneficiary for both parts.

This e-psychology platform was based on modern informative and communicative tools and services as is the case in many modern e-learning platforms. More particularly, it was designed and developed very meticulously in an effort to provide an innovative platform that was user-centered, user-friendly, modular and flexible. In addition, the aim was to exploit in full all the capabilities of contemporary Internet technologies for the benefit of both therapists and patients but mostly in an effort to primarily cover as much as possible the psychological needs (regardless of the field) of the aforementioned users of the platform.

\section{References}

1. Brusilovski, P., Eklund, J., Schwarz, E.: Web-Based Education for All: A Tool For Development Adaptive Courseware. In: 7th International WWW Conference, pp. 291-300. Elsevier, Amsterdam (1998)

2. Durm, R., Duval, E., Verhoeven, B., Cardinaels, K., Olivie, H.: Extending the ARIADNE Web-Based Learning Environment. In: World Conference on Educational Multimedia, Hypermedia and Telecommunications, pp. 1932-1937 (2001)

3. García, V., Ahumada, L., Hinkelman, J., Muñoz, R., Quezada, J.: Psychology over the Internet: On-Line Experiences. In: 29th InterAmerican Congress of Psychology, pp. 29-33 (2004)

4. Plant, R., Howes, A., Hammond, N., Trapp, A.: A Virtual Psychology Lab: A Look Back Over the Last Three Years. In: Computers in Psychology Conference (2000)

5. Marks, I., Shaw, S., Parkin, R.: Computer-Aided Treatments of Mental Health Problems. In: Clinical Psychology: Science and Practice, pp. 151-170 (1998)

6. Kenwright, M.: Computer-Aided Self-Treatment for Phobias. In: Computers in Psychology Conference (2000) 
7. Riva, G., Molinari, E., Vincelli, F.: Interaction and Presence in the Clinical Relationship: Virtual Reality (VR) as a Communicative Medium between Patient and Therapist. In: IEEE Transactions on Information Technology in Biomedicine, pp. 198-205 (2002)

8. Vincelli, F.: From Imagination to Virtual Reality: The Future of Clinical Psychology. In: CyberPsychology and Behavior, pp. 241-248 (1999)

9. Vincelli, F., Riva, G.: Virtual Reality: A New Tool for Panic Disorder Therapy. In: Expert Review of Neurotherapeutics, pp. 377-383 (2002)

10. Gonzalez, G.: Bilingual Telephone-Assisted Computerized Speech Recognition Interviewing: Automated Screening of Depression Symptoms. In: Computers in Psychology Conference (2000)

11. Richter, T., Naumann, G.: Computer-Based Assessment of Reading Skills. In: Computers in Psychology Conference (2000)

12. MacRae, A.: Multimedia Statistics Course on CD: The First Year's Experience. In: Computers in Psychology Conference (2000)

13. Morris, E., Scanlon, E., Joiner, R.: Evaluating Multimedia Resources for Teaching Statistics to Psychology Undergraduates. In: Computers in Psychology Conference (2000)

14. Gomella, L.: The Wild, Wild Web: Resources for Counseling Patients with Prostate Cancer in the Information Age. In: Semin Urologic Oncol, pp. 167-171 (2000)

15. Eysenbach, G., Powell, J., Kuss, O.: Empirical Studies Assessing the Quality of Health Information for Consumers on the World Wide Web: A Systematic Review. JAMA 287, 2691-2700 (2002)

16. Matthews, S., Camacho, A., Mills, P., et al.: The Internet for Medical Information About Cancer: Help or Hindrance? Psychosomatics 44, 100-103 (2003)

17. Fogel, J., Albert, S., Schnabel, F.: Quality of Health Information on the Internet. JAMA 286, 2093-2095 (2001)

18. Fogel, J.: Internet Breast Health Information: Use and Coping Among Women with Breast Cancer. CyberPsychology \& Behavior 7, 59-63 (2004)

19. Carlson, N., Buskist, W.: Psychology: The Science of Behavior. Allyn and Bacon, Inc. (1997) 